\title{
Linx
}

Revue des linguistes de l'université Paris X Nanterre

47 | 2002

Du sens au sens

\section{Michel Galmiche, peinture, sémantique et réalité}

Michel Galmiche, painting and reality

\section{Daniel Delas}

\section{OpenEdition}

\section{Journals}

Édition électronique

URL : http://journals.openedition.org/linx/596

DOI : $10.4000 /$ linx.596

ISSN : 2118-9692

\section{Éditeur}

Presses universitaires de Paris Nanterre

\section{Édition imprimée}

Date de publication : 1 décembre 2002

Pagination : 175-179

ISSN : 0246-8743

\section{Référence électronique}

Daniel Delas, « Michel Galmiche, peinture, sémantique et réalité », Linx [En ligne], 47 | 2002, mis en ligne le 01 juin 2003, consulté le 02 mai 2019. URL : http://journals.openedition.org/linx/596 ; DOI : 10.4000/linx.596 


\title{
Michel Galmiche, peinture, sémantique et réalité
}

\author{
Daniel Delas, Université de Cergy-Pontoise
}

Qu'on ne s'attende pas un traité sur cette importante question. Ce sont ici quelques réflexions qui sont nées de la fréquentation de quelques cuvres (3) de Michel que je vois chaque jour sur les murs de chez moi. A force d'être là, elles se sont en quelque sorte constituées en un lieu par où passent et repassent les interrogations du poéticien vagabond que je suis.

\section{Des arts et de la représentation}

La représentation du réel a-t-elle des artistes plus spécialisés que d'autres ?

Les musiciens ne se préoccupent plus guère - heureusement d'ailleurs! eu égard au faible niveau des œuvres musicales dites réalistes - de représenter ${ }^{1}$ directement la réalité. En d'autres termes ils ne cherchent guère à la reproduire.

Le peintre (désigné ici comme le parangon de l'artiste du visuel mais auquel il faudrait adjoindre le photographe ${ }^{2}$ ), le poète (désigné ici, dans notre société occidentale post-romantique, comme le professionnel par excellence du langage verbal artistique mais qui existe sous bien d'autres espèces littéraires, romancier, dramaturge, scénariste, essayiste, etc.) et le linguiste (terme à prendre dans l'acception la plus générale et à étendre à des poètes-poéticiens comme Paul Valéry, Francis Ponge ou Yves Bonnefoy aussi bien qu'à certains philosophes) sont parties prenantes d'une définition extensive du terme représenter. Le peintre et le poète utilisent l'un comme l'autre, pour représenter le monde et l'homme dans le monde, un langage que caractérise son historicité et sa subjectivité. Historicité que ne se ramène pas seulement au contexte historique mais inclut les représentations du langage et du monde, subjectivité qui

\footnotetext{
${ }^{1}$ Il ne saurait être question de définir définitivement ce terme d'entrée de jeu puisque c'est sa définition même qui est en question dans cette réflexion.

${ }^{2}$ Et dans sa mouvance le cinéaste, mais là, les choses se compliquent et on laissera le cinéma de côté dans cette modeste réflexion.
} 
n'est pas celle seulement de l'individu artiste mais relève de l'action continue d'un sujet de l'écriture.

Tantôt par exemple «ce qui se conçoit bien s'énonce clairement», tantôt ce qu'on laisse s'énoncer « automatiquement» signifie pleinement et profondément, dans les hasards de son énonciation même. Tantôt la tête affirme guider le pinceau, tantôt c'est le corps qui s'imprime directement sur le papier. Chaque époque prise dans son historicité et dans la volonté de s'affranchir de l'académisme et des stéréotypes historiques dominants, ne se prive pas de surcroit de réécrire le passé en fonction de la poétique qu'elle élabore au confluent des deux forces présentes dans toute poétique représentative du monde : la matière de l'art et la règle de l'art.

Les couleurs qu'utilise le peintre ont en soi autant et aussi peu de valeur représentative que les sons ou les propriétés phonétiques des séquences que met en place le poète, en ce sens qu'en la double absence d'une part d'un réel transhistorique fixe auquel renverraient toutes les formes d'art au cours des siècles ${ }^{3}$, d'autre part de techniques fixes qui donneraient les mêmes couleurs à toutes les époques et dans toutes les civilisations ou qui figeraient les langues dans un capital de sonorités et de mots solidifié à jamais, la poétique de la couleur ou des mots sonores doit se réinventer sans cesse et partout, la compétence représentative n'apparaissant pas comme une simple question de technique mais comme une aptitude à bien questionner tel réel à telle époque. Les comparaisons musicales dont on se sert parfois pour décrire l'effet d'un poème ou d'un tableau, - symphonie des couleurs/musique des mots -, ne révèlent-elles pas d'ailleurs que c'est bien cette même énigme qui de siècles en siècles interpelle sans cesse le discours sur le peintre et le poète?

Joindre à l'artiste stricto sensu, au créateur incontesté qu'est le peintre ou le poète, le poéticien ou le sémanticien ${ }^{4}$ ne va pas sans soulever de vives réserves dans la doxa dominante, tant est toujours prégnant aujourd'hui le stéréotype du génie a-historique, voire transcendantal, classique aussi bien que romantique, considéré comme totalement créateur, premier, opposé à celui de l'intellectuel, du critique ou du professeur, souvent considéré par les partisans d'une conception sacralisante de l'artiste, comme un serviteur, un simple commentateur qui se contente a posteriori d'établir certaines relations internes et externes de l'œuvre. Dualisme aveugle et sans valeur qui ne mérite pas qu'on le combatte longuement. Il est impossible aujourd'hui de dissocier théorie et pratique, les sciences humaines, quelque discutables que soient les délimitations de leurs frontières, ont apporté des connaissances sur l'homme qui obligent à associer leurs porte-parole et leurs travaux à toute réflexion sur la représentation du monde par l'art. C'est dans cette relation même de la réflexion et des pratiques que pourra s'élancer l'amour de l'art.

Certains poètes et certains grammairiens font d'ailleurs le même travail : ils soupèsent et combinent à l'infini des bouts du matériau qui sert à représenter, en tentant d'accéder à des hypothèses systémiques. Qu'ils fassent le même travail, c'est ce que révèle le fait qu'ils sont souvent l'un et l'autre considérés dans leur domaine comme les maîtres d'un secret des mots, le sémanticien étant pour ceux qui savent

\footnotetext{
${ }^{3}$ Croyance aristotélicienne qui donne à représenter le sens d’une étymologie : re-présenter : présenter un réel antérieur ET supérieur.

${ }^{4}$ On peut aussi l'appeler grammairien, essayiste.
} 
trois ou quatre mots de sciences du langage une espèce supérieure (et donc suspecte!) de linguiste, le poète étant vu comme un maitre du secret des mots ${ }^{5}$.

Le linguiste Michel Galmiche, sémanticien s'il en fut, faisait partie de cette phalange au double visage, dans le bataillon non des créateurs-poètes mais en tant que créateur-peintre. Une question intéressante à poser s'impose : quel lien peut-il exister entre le genre de peinture qui fut le sien et la sémantique qu'il illustra ? Que le lecteur se rassure, je n'ai pas la réponse et je ne lui propose que de divaguer quelques instants sur cette question.

\section{L'hyperréalisme entre naturel et artificiel}

Pour ce que je connais de son œuvre, Michel Galmiche a choisi pour ses tableaux majeurs une forme particulière de représentation du réel, proche de ce qu'on appelle parfois l'hyperréalisme: rues, cafés, intérieurs, ponts, carrefours, lieux montants ou descendants sont représentés minutieusement, dans la rutilance ou la matité de leurs détails, avec des glacis soignés qui leur donnent l'épaisseur qu'ils méritent. Des gens aussi certes mais sous forme de silhouettes massives projetées dans le monde, là, en attente.

Première constatation: Galmiche n'a pas choisi un mode non figuratif d'expression. Peut-être parce que trouver son mode non-figuratif d'expression est une cheminement plus ambitieux, qui aboutit difficilement et demande un investissement temporel total, un long travail de torsion et d'abstraction du mode figuratif d'expression. Il faut beaucoup " y croire », épaissir les pâtes, introduire des matériaux bruts, provoquer par les couleurs, des matériaux nouveaux, des mises en scène violentes ou sarcastiques, etc. Ce qui mène à s'immerger en quelque sorte dans le monde qu'on repense, pour chercher de l'intérieur comment agresser efficacement réel et social de sorte à les fissurer et à exhiber ce qu'on pense être sa vérité bic et nunc ${ }^{6}$. Certes Soulages n'est ni Sam Francis ni Pollock, mais chacun a son monde, est dans son monde.

Mais en choisissant de peindre selon un mode hyperréaliste du figuratif, Galmiche se détache aussi du réalisme-naturaliste-régionaliste académique des peintres du dimanche mais ne s'installe pas dans la rupture totale mais plutôt dans une sorte d'entre-deux, à la frontière du réalisme «naturel » et de l'artificiel. Dans l'introduction à son livre sur la théorie de R. Montague ${ }^{7}$, Michel cite celui-ci pour une phrase «provocatrice» qu'il admire : «Je regrette l'idée qu'il existe une différence théorique importante entre les langages formels et les langues naturelles ». Ce regret laissant entendre qu'une osmose ou une synthèse entre formel et naturel ouvrirait le monde à

\footnotetext{
${ }^{5}$ Saussure expliquait les anagrammes par un savoir secret du poète.

${ }^{6}$ On peut penser à l'entreprise extraordinaire de Dubuffet à l'époque de l'Hourloupe (à partir de 1963), s'installant dans une «maison» qu'il a bâtie de ses propres mains, avec ses propres outils. Il est désormais dans la demeure de sa création, déployant son logos et non le logos de la tradition. Voir Marcel Paquet, Dubuffet, Nouvelles Editions Françaises/Casterman, p. 157 sqq.

7 Sémantique linguistique et logique. Un exemple : la théorie de R. Montague, PUF, 1991, p. 18
} 
une sorte de perfection représentative. Ne serait-ce pas ce que permet de réaliser, à sa manière et mutatis mutandis, la peinture hyperréaliste?

Voyons ce qu'il en est à partir d'un exemple concret.

Le bon vieux réalisme-naturaliste du $19^{\mathrm{e}}$ ne se pratique plus guère officiellement, mais il hante les salons d'amateurs de peinture. Donnons-en un exemple peu connu qui distraira le lecteur, celui d'Almeida junior (1850-1899). Jeune et brillant artiste brésilien, il bénéficia d'une bourse impériale pour compléter sa formation à Paris auprès de Cabanel avant de retourner à Sao Paulo. Sa renommée s'affirme dans deux domaines, une peinture à la gloire de la femme, où il représente sa maitresse, femme d'un riche fazendeiro, dans des situations osées, laissant deviner la silhouette du cocu derrière la porte et la peinture de genre, celle des paysans brésiliens, exécutée avec un grand luxe de détails, dans le plus pur style de ce qu'on qualifiera d'indigéniste plus tard. Dans deux de ces tableaux paysans, le caipeira aiguise tantôt un long couteau, tantôt une hache. Et même dans Amolação interrompida, le paysan, la hache à la main, se redresse comme pour passer à l'action. Or peu après, le peintre fut assassiné par le mari trompé. Lequel fut acquitté. Voilà du réalisme saignant. Un réalisme qui provoque la vie individuelle, qui court après une réalité naturelle, celle du fait-divers et qui " prouve » sa vérité quand, comme on dit, le réel rattrape la fiction, en une histoire bien croustillante.

Ce réalisme est évidemment narratif et donc fictionnel. Autrement dit soumis aux contraintes d'une écriture d'art obstinée à obtenir l'effet de réel, le grand frisson (ici obtenu au delà de toute espérance !).

L'hyperréalisme n'est ni narratif ni donc fictionnel. Il ne cherche en rien à donner l'illusion du mouvement et du continu de la vie. Il peut tout au plus s'associer à une image arrêtée. Il n'est pas particulièrement proche de la photographie toutefois car celle-ci n'arrête pas tant l'image qu'elle n'en fournit un instant (un instantané). Le tableau hyperréaliste souligne toutes les lignes et sépare bien les couleurs du réel qu'il représente pour en faire ressortir artificiellement une sorte d'essence pure. Finalement, en poussant le raisonnement jusqu'au bout, on pourrait soutenir qu'il est un antiréalisme dans la mesure où un réalisme épuré a perdu ce qui justement fait l'épaisse vérité du réel. Le début du $20^{\mathrm{e}}$ siècle met en crise en Europe le mode d'appréhension direct du réel, et non sans liens avec le surréalisme, développe un courant qu'on peut appeler factographique, produisant en guise de littérature des "proses sans intrigue », constituant un vaste champ transgénérique appelé otcherk dans la Russie du 19ème. Les tableaux de Magritte ou de Delvaux situent des personnages désincarnés dans des décors stylisés.

La peinture de Galmiche me semble pouvoir être approchée par ce terme d'otcherk en ce qu'elle n'est ni narrative ni artistique ${ }^{8}$, mais bien plutôt documentaire (ce sont des lieux réels (tel café dans telle rue)), discontinue (elle ne suggère ni un

8 «Ce mot combine la signification d'esquisse ou d'étude (au sens que le terme a en peinture) et celle, quasi scientifique, d'étude physiologique.(...) L'otcherk n'abolit ni la figure du narrateur, ni la tension événementielle, ni le jeu stylistique. Mais, au lieu de dissimuler ses thèmes à l'abri du procédé narratif, il les explicite sous forme de problèmes «vrais », extérieurs à son déploiement. » (Leonid Heller, «Remarques sur la littérature factographique en Russie », Communications $\mathrm{n}^{\circ} 71$, « Le parti-pris du document », Seuil, 2001, p. 47) 
avant ni un après) et figurative d'un réel en quelque sorte artificiel, si l'on donne à ce dernier terme, le sens d'« épuré de l'épaisseur opaque du réel»). Les formalistes russes ont parlé de la véritable «biographie de l'objet» que permettrait un récit déconnecté de l'événementiel et ainsi proche d'une vérité défocalisée, captant l'air du temps. C'est, me semble-t-il, le cas chez Galmiche, ses motifs, hommes ou choses, sont objectivés dans une attente dont ni l'auteur ni les personnages ni les choses ne savent l'issue. Comme dans les tableaux de Hopper mais sans aucune dimension psychologique ou sociale. Qu'attend le monde galmichien, en sa respiration lourde et lente?

Ainsi se trouve concrétisé un langage pictural à mi-chemin entre l'artificiel et le naturel, le plus près possible d'une vérité dont la pureté est à la fois la caution euphorisante et la perte douloureuse.

Dans l'attente, pour le meilleur et pour le pire.

Daniel DELAS

Université de Cergy-Pontoise

5 , rue Broussais

75014 PARIS

DanielDelas@aol.com 